\title{
Ideals of BCI-algebras and their Properties
}

\author{
Chenglong $\mathrm{Wu}$ \\ Qingdao University of Science \\ and Technology \\ China
}

\author{
Yuzhong Ding \\ Qingdao University of Science \\ and Technology \\ China
}

Summary. In this article three classes of ideals are discussed: associative ideals, commutative ideals, implicative ideals and positive implicative ideals, and their elementary properties. Some of their properties and the relationships between them have not been proven yet, and will be completed in the following article.

MML identifier: $\underline{\text { BCIIDEAL, version: } \underline{7.8 .10 \quad 4.99 .1005}}$

The papers [4], [1], [2], and [3] provide the terminology and notation for this paper.

\section{Preliminaries}

For simplicity, we use the following convention: $X$ denotes a BCI-algebra, $X_{1}$ denotes a non empty subset of $X, A, I$ denote ideals of $X, x, y, z$ denote elements of $X$, and $a$ denotes an element of $A$.

The following four propositions are true:

(1) For all elements $x, y, z, u$ of $X$ such that $x \leq y$ holds $u \backslash(z \backslash x) \leq u \backslash(z \backslash y)$.

(2) For all elements $x, y, z, u$ of $X$ holds $x \backslash(y \backslash z) \backslash(x \backslash(y \backslash u)) \leq z \backslash u$.

(3) For all elements $x, y, z, u, v$ of $X$ holds $x \backslash(y \backslash(z \backslash u)) \backslash(x \backslash(y \backslash(z \backslash v))) \leq$ $v \backslash u$.

(4) For all elements $x, y$ of $X$ holds $0_{X} \backslash(x \backslash y) \backslash(y \backslash x)=0_{X}$.

Let us consider $X$ and let $a$ be an element of $X$. The initial section of $a$ is defined by:

(Def. 1) The initial section of $a=\{x \in X: x \leq a\}$. 
The following propositions are true:

(5) If $x \leq a$, then $x \in A$.

(6) For all elements $x, a, b$ of AtomSet $X$ such that $x$ is an element of BranchV $b$ holds $a \backslash x=a \backslash b$.

(7) For every element $a$ of $X$ and for all elements $x, b$ of AtomSet $X$ such that $x$ is an element of BranchV $b$ holds $a \backslash x=a \backslash b$.

(8) The initial section of $a \subseteq A$.

(9) If AtomSet $X$ is an ideal of $X$, then for every element $x$ of BCK-part $X$ and for every element $a$ of AtomSet $X$ such that $x \backslash a \in$ AtomSet $X$ holds $x=0_{X}$.

(10) If AtomSet $X$ is an ideal of $X$, then AtomSet $X$ is a closed ideal of $X$.

Let us consider $X, I$. We say that $I$ is positive if and only if:

(Def. 2) Every element of $I$ is positive.

Next we state three propositions:

(11) Let $X$ be a BCK-algebra and $A, I$ be ideals of $X$. Then $A \cap I=\left\{0_{X}\right\}$ if and only if for every element $x$ of $A$ and for every element $y$ of $I$ holds $x \backslash y=x$.

(12) For every associative BCI-algebra $X$ holds every ideal of $X$ is closed.

(13) For every BCI-algebra $X$ and for every ideal $A$ of $X$ such that $X$ is quasi-associative holds $A$ is closed.

\section{Definitions of Associative Ideals}

Let $X$ be a BCI-algebra and let $I_{1}$ be an ideal of $X$. We say that $I_{1}$ is associative if and only if:

(Def. 3) $\quad 0_{X} \in I_{1}$ and for all elements $x, y, z$ of $X$ such that $x \backslash(y \backslash z), y \backslash z \in I_{1}$ holds $x \in I_{1}$.

Let $X$ be a BCI-algebra. One can verify that there exists an ideal of $X$ which is associative.

Let $X$ be a BCI-algebra. A non empty subset of $X$ is said to be an associativeideal of $X$ if:

(Def. 4) $\quad 0_{X} \in$ it and for all elements $x, y, z$ of $X$ such that $x \backslash y \backslash z, y \backslash z \in$ it holds $x \in$ it.

We now state four propositions:

(14) If $X_{1}$ is an associative-ideal of $X$, then $X_{1}$ is an ideal of $X$.

(15) $\quad I$ is an associative-ideal of $X$ iff for all $x, y, z$ such that $x \backslash y \backslash z \in I$ holds $x \backslash(y \backslash z) \in I$.

(16) If $I$ is an associative-ideal of $X$, then for every element $x$ of $X$ holds $x \backslash\left(0_{X} \backslash x\right) \in I$. 
(17) If for every element $x$ of $X$ holds $x \backslash\left(0_{X} \backslash x\right) \in I$, then $I$ is a closed ideal of $X$.

Let $X$ be a BCI-algebra. A non empty subset of $X$ is said to be a $p$-ideal of $X$ if:

(Def. 5) $0_{X} \in$ it and for all elements $x, y, z$ of $X$ such that $x \backslash z \backslash(y \backslash z), y \in$ it holds $x \in$ it.

We now state several propositions:

(18) If $X_{1}$ is a $p$-ideal of $X$, then $X_{1}$ is an ideal of $X$.

(19) For all $X, I$ such that $I$ is a $p$-ideal of $X$ holds BCK-part $X \subseteq I$.

(20) BCK-part $X$ is a $p$-ideal of $X$.

(21) $I$ is a $p$-ideal of $X$ iff for all $x, y$ such that $x \in I$ and $x \leq y$ holds $y \in I$.

(22) $I$ is a $p$-ideal of $X$ iff for all $x, y, z$ such that $x \backslash z \backslash(y \backslash z) \in I$ holds $x \backslash y \in I$.

\section{Definitions of Commutative Ideals}

Let $X$ be a BCK-algebra and let $I_{1}$ be an ideal of $X$. We say that $I_{1}$ is commutative if and only if:

(Def. 6) For all elements $x, y, z$ of $X$ such that $x \backslash y \backslash z, z \in I_{1}$ holds $x \backslash(y \backslash(y \backslash x)) \in$ $I_{1}$.

Let $X$ be a BCK-algebra. One can verify that there exists an ideal of $X$ which is commutative.

Next we state two propositions:

(23) For every BCK-algebra $X$ holds BCK-part $X$ is a commutative ideal of $X$.

(24) Let $X$ be a BCK-algebra. Suppose $X$ is a $p$-semisimple BCI-algebra. Then $\left\{0_{X}\right\}$ is a commutative ideal of $X$.

In the sequel $X$ denotes a BCK-algebra.

One can prove the following proposition

(25) BCK-part $X=$ the carrier of $X$.

In the sequel $X$ denotes a BCI-algebra.

We now state several propositions:

(26) If for every BCI-algebra $X$ and for all elements $x, y$ of $X$ holds $x \backslash y \backslash y=$ $x \backslash y$, then the carrier of $X=$ BCK-part $X$.

(27) If for every BCI-algebra $X$ and for all elements $x, y$ of $X$ holds $x \backslash(y \backslash x)=$ $x$, then the carrier of $X=\mathrm{BCK}$-part $X$.

(28) If for every BCI-algebra $X$ and for all elements $x, y$ of $X$ holds $x \backslash(x \backslash y)=$ $y \backslash(y \backslash x)$, then the carrier of $X=$ BCK-part $X$. 
(29) If for every BCI-algebra $X$ and for all elements $x, y, z$ of $X$ holds $(x \backslash$ $y) \backslash y=x \backslash z \backslash(y \backslash z)$, then the carrier of $X=$ BCK-part $X$.

(30) If for every BCI-algebra $X$ and for all elements $x, y$ of $X$ holds $x \backslash y \backslash$ $(y \backslash x)=x \backslash y$, then the carrier of $X=$ BCK-part $X$.

(31) If for every BCI-algebra $X$ and for all elements $x, y$ of $X$ holds $x \backslash y \backslash$ $(x \backslash y \backslash(y \backslash x))=0_{X}$, then the carrier of $X=$ BCK-part $X$.

(32) For every BCK-algebra $X$ holds the carrier of $X$ is a commutative ideal of $X$.

In the sequel $X$ denotes a BCK-algebra and $I$ denotes an ideal of $X$.

One can prove the following propositions:

(33) $I$ is a commutative ideal of $X$ iff for all elements $x, y$ of $X$ such that $x \backslash y \in I$ holds $x \backslash(y \backslash(y \backslash x)) \in I$.

(34) Let $I, A$ be ideals of $X$. Suppose $I \subseteq A$ and $I$ is a commutative ideal of $X$. Then $A$ is a commutative ideal of $X$.

(35) Every ideal of $X$ is a commutative ideal of $X$ iff $\left\{0_{X}\right\}$ is a commutative ideal of $X$.

(36) $\left\{0_{X}\right\}$ is a commutative ideal of $X$ iff $X$ is a commutative BCK-algebra.

(37) $X$ is a commutative BCK-algebra iff every ideal of $X$ is a commutative ideal of $X$.

(38) $\left\{0_{X}\right\}$ is a commutative ideal of $X$ iff every ideal of $X$ is a commutative ideal of $X$.

In the sequel $I$ denotes an ideal of $X$.

One can prove the following propositions:

(39) For all elements $x, y$ of $X$ such that $x \backslash(x \backslash y) \in I$ holds $x \backslash(x \backslash y \backslash(x \backslash y \backslash x))$, $y \backslash(y \backslash x) \backslash x, y \backslash(y \backslash x) \backslash(x \backslash y) \in I$.

(40) $\left\{0_{X}\right\}$ is a commutative ideal of $X$ iff for all elements $x, y$ of $X$ holds $x \backslash(x \backslash y) \leq y \backslash(y \backslash x)$.

(41) $\left\{0_{X}\right\}$ is a commutative ideal of $X$ iff for all elements $x, y$ of $X$ holds $x \backslash y=x \backslash(y \backslash(y \backslash x))$.

(42) $\left\{0_{X}\right\}$ is a commutative ideal of $X$ iff for all elements $x, y$ of $X$ holds $x \backslash(x \backslash y)=y \backslash(y \backslash(x \backslash(x \backslash y)))$.

(43) $\quad\left\{0_{X}\right\}$ is a commutative ideal of $X$ iff for all elements $x, y$ of $X$ such that $x \leq y$ holds $x=y \backslash(y \backslash x)$.

(44) Suppose $\left\{0_{X}\right\}$ is a commutative ideal of $X$. Then

(i) for all elements $x, y$ of $X$ holds $x \backslash y=x$ iff $y \backslash(y \backslash x)=0_{X}$,

(ii) for all elements $x, y$ of $X$ such that $x \backslash y=x$ holds $y \backslash x=y$,

(iii) for all elements $x, y, a$ of $X$ such that $y \leq a$ holds $a \backslash x \backslash(a \backslash y)=y \backslash x$,

(iv) for all elements $x, y$ of $X$ holds $x \backslash(y \backslash(y \backslash x))=x \backslash y$ and $x \backslash y \backslash(x \backslash y \backslash x)=$ $x \backslash y$, and 
(v) for all elements $x, y, a$ of $X$ such that $x \leq a$ holds $(a \backslash y) \backslash(a \backslash y \backslash(a \backslash x))=$ $a \backslash y \backslash(x \backslash y)$.

(45) Every ideal of $X$ is a commutative ideal of $X$ iff for all elements $x, y$ of $X$ holds $x \backslash(x \backslash y) \leq y \backslash(y \backslash x)$.

(46) Every ideal of $X$ is a commutative ideal of $X$ iff for all elements $x, y$ of $X$ holds $x \backslash y=x \backslash(y \backslash(y \backslash x))$.

(47) Every ideal of $X$ is a commutative ideal of $X$ iff for all elements $x, y$ of $X$ holds $x \backslash(x \backslash y)=y \backslash(y \backslash(x \backslash(x \backslash y)))$.

(48) Every ideal of $X$ is a commutative ideal of $X$ iff for all elements $x, y$ of $X$ such that $x \leq y$ holds $x=y \backslash(y \backslash x)$.

(49) Suppose every ideal of $X$ is a commutative ideal of $X$. Then

(i) for all elements $x, y$ of $X$ holds $x \backslash y=x$ iff $y \backslash(y \backslash x)=0_{X}$,

(ii) for all elements $x, y$ of $X$ such that $x \backslash y=x$ holds $y \backslash x=y$,

(iii) for all elements $x, y, a$ of $X$ such that $y \leq a$ holds $a \backslash x \backslash(a \backslash y)=y \backslash x$,

(iv) for all elements $x, y$ of $X$ holds $x \backslash(y \backslash(y \backslash x))=x \backslash y$ and $x \backslash y \backslash(x \backslash y \backslash x)=$ $x \backslash y$, and

(v) for all elements $x, y, a$ of $X$ such that $x \leq a$ holds $(a \backslash y) \backslash(a \backslash y \backslash(a \backslash x))=$ $a \backslash y \backslash(x \backslash y)$.

\section{Definitions of Implicative Ideals and Positive Implicative Ideals}

Let $X$ be a BCK-algebra. A non empty subset of $X$ is said to be an implicativeideal of $X$ if:

(Def. 7) $\quad 0_{X} \in$ it and for all elements $x, y, z$ of $X$ such that $x \backslash(y \backslash x) \backslash z, z \in$ it holds $x \in$ it.

In the sequel $X$ denotes a BCK-algebra and $I$ denotes an ideal of $X$.

Next we state the proposition

(50) $I$ is an implicative-ideal of $X$ iff for all elements $x, y$ of $X$ such that $x \backslash(y \backslash x) \in I$ holds $x \in I$.

Let $X$ be a BCK-algebra. A non empty subset of $X$ is said to be a positiveimplicative-ideal of $X$ if:

(Def. 8) $0_{X} \in$ it and for all elements $x, y, z$ of $X$ such that $x \backslash y \backslash z, y \backslash z \in$ it holds $x \backslash z \in$ it.

We now state several propositions:

(51) $I$ is a positive-implicative-ideal of $X$ if and only if for all elements $x, y$ of $X$ such that $x \backslash y \backslash y \in I$ holds $x \backslash y \in I$.

(52) Suppose that for all elements $x, y, z$ of $X$ such that $x \backslash y \backslash z, y \backslash z \in I$ holds $x \backslash z \in I$. Let $x, y, z$ be elements of $X$. If $x \backslash y \backslash z \in I$, then $x \backslash z \backslash(y \backslash z) \in I$.

(53) Suppose that for all elements $x, y, z$ of $X$ such that $x \backslash y \backslash z \in I$ holds $x \backslash z \backslash(y \backslash z) \in I$. Then $I$ is a positive-implicative-ideal of $X$. 
(54) $I$ is a positive-implicative-ideal of $X$ if and only if for all elements $x, y$, $z$ of $X$ such that $x \backslash y \backslash z, y \backslash z \in I$ holds $x \backslash z \in I$.

(55) $I$ is a positive-implicative-ideal of $X$ if and only if for all elements $x, y$, $z$ of $X$ such that $x \backslash y \backslash z \in I$ holds $x \backslash z \backslash(y \backslash z) \in I$.

(56) Let $I, A$ be ideals of $X$. Suppose $I \subseteq A$ and $I$ is a positive-implicativeideal of $X$. Then $A$ is a positive-implicative-ideal of $X$.

(57) Suppose $I$ is an implicative-ideal of $X$. Then $I$ is a commutative ideal of $X$ and a positive-implicative-ideal of $X$.

\section{REFERENCES}

[1] Yuzhong Ding. Several classes of BCI-algebras and their properties. Formalized Mathematics, 15(1):1-9, 2007.

[2] Yuzhong Ding and Zhiyong Pang. Congruences and quotient algebras of BCI-algebras. Formalized Mathematics, 15(4):175-180, 2007.

[3] Tao Sun, Dahai Hu, and Xiquan Liang. Several classes of BCK-algebras and their properties. Formalized Mathematics, 15(4):237-242, 2007.

[4] Zinaida Trybulec. Properties of subsets. Formalized Mathematics, 1(1):67-71, 1990.

Received March 3, 2008 